\title{
Resistive switching effects in single metallic tunneling junction with nanometer-scale gap
}

\section{AUTHOR(S):}

Mizukami, Takahiro; Miyato, Yuji; Kobayashi, Kei; Matsushige, Kazumi; Yamada, Hirofumi

\section{CITATION:}

Mizukami, Takahiro ...[et al]. Resistive switching effects in single metallic tunneling junction with nanometer-scale gap. APPLIED PHYSICS LETTERS 2011, 98(8): 083120.

\section{ISSUE DATE:}

2011-02

URL:

http://hdl.handle.net/2433/160628

\section{RIGHT:}

Copyright 2011 American Institute of Physics. This article may be downloaded for personal use only. Any other use requires prior permission of the author and the American Institute of Physics. The following article appeared in APPLIED PHYSICS LETTERS 98, 083120 (2011) and may be found at http://link.aip.org/link/?apl/98/083120 


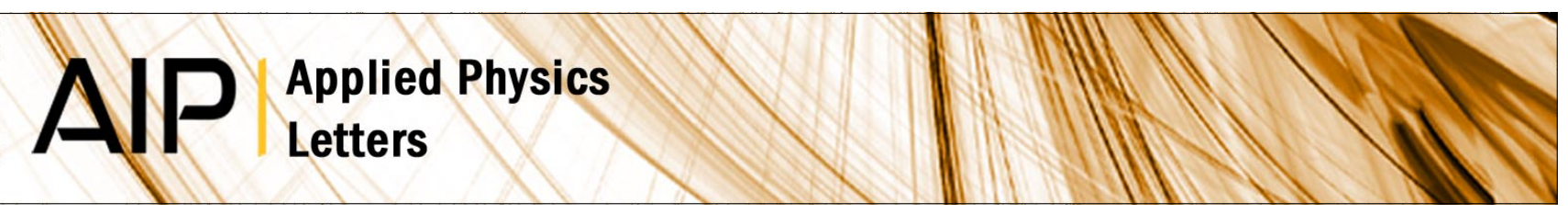

\section{Resistive switching effects in single metallic tunneling junction with nanometer-scale gap}

Takahiro Mizukami, Yuji Miyato, Kei Kobayashi, Kazumi Matsushige, and Hirofumi Yamada

Citation: Appl. Phys. Lett. 98, 083120 (2011); doi: 10.1063/1.3559612

View online: http://dx.doi.org/10.1063/1.3559612

View Table of Contents: http://apl.aip.org/resource/1/APPLAB/v98/i8

Published by the American Institute of Physics.

\section{Related Articles}

Pinned interface dipole-induced tunneling electroresistance in ferroelectric tunnel junctions: A theoretical investigation

J. Appl. Phys. 112, 054104 (2012)

Current-voltage characteristics and ON/OFF ratio in ferroelectric tunnel junctions

J. Appl. Phys. 112, 054102 (2012)

Observation of fluctuation-induced tunneling conduction in micrometer-sized tunnel junctions

AlP Advances 2, 032155 (2012)

Conductivity mapping of nanoparticles by torsional resonance tunneling atomic force microscopy

Appl. Phys. Lett. 101, 083107 (2012)

Zero-bias anomaly in one-dimensional ultrathin metallic nanowires

AlP Advances 2, 032143 (2012)

\section{Additional information on Appl. Phys. Lett.}

Journal Homepage: http://apl.aip.org/

Journal Information: http://apl.aip.org/about/about_the_journal

Top downloads: http://apl.aip.org/features/most_downloaded

Information for Authors: http://apl.aip.org/authors

\section{ADVERTISEMENT}

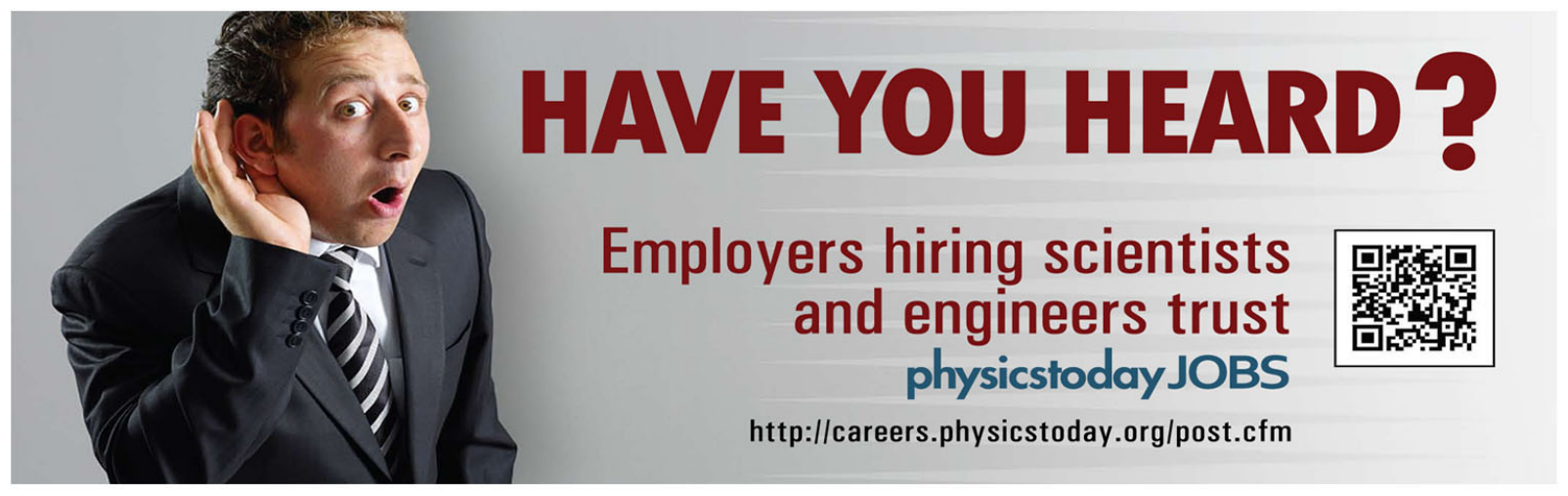




\title{
Resistive switching effects in single metallic tunneling junction with nanometer-scale gap
}

\author{
Takahiro Mizukami, ${ }^{1}$ Yuji Miyato, ${ }^{1}$ Kei Kobayashi, ${ }^{2}$ Kazumi Matsushige, ${ }^{1}$ and \\ Hirofumi Yamada ${ }^{1, a)}$ \\ ${ }^{1}$ Department of Electronic Science and Engineering, Kyoto University, Katsura, Nishikyo, \\ Kyoto 615-8510, Japan \\ ${ }^{2}$ Office of Society-Academia Collaboration for Innovation, Kyoto University, Katsura, Nishikyo, \\ Kyoto 615-8520, Japan
}

(Received 5 January 2011; accepted 4 February 2011; published online 25 February 2011)

\begin{abstract}
We fabricated a single tunneling junction with a nanometer-scale gap between Pt electrodes. We found that the gap distance became smaller after a current sweep, which was presumably caused by the migration of the Pt atoms at the anode. The junction showed a reproducible negative differential resistance characteristic after reduction in the gap. The junction also showed resistive switching characteristics with a resistance ratio of over 100 by applying voltage of different waveforms. The tunneling area and gap distance for on/off-state were quantitatively estimated by fitting the measured characteristics to the simple model as $100 \mathrm{~nm}^{2}$ and $0.8 / 1.2 \mathrm{~nm}$, respectively. (c) 2011 American Institute of Physics. [doi:10.1063/1.3559612]
\end{abstract}

A wide variety of resistive switching devices are currently proposed for future nonvolatile memory systems. They include such as calcogenide phase-change memory devices, ${ }^{1}$ resistive random access memory based on metal oxide, ${ }^{2}$ atomic switches of the ionic salts, ${ }^{3,4}$ and molecular switches. ${ }^{5}$

For the realization of ultrahigh density memory devices, the structure of the individual memory cell should be as simple as possible. Recently, Naitoh and co-workers ${ }^{6-10}$ reported a negative differential resistance (NDR) characteristics in metallic electrode junctions with a nanometer-scale gap. They also found that the current-voltage (I-V) curves of those nanoscale-gap junctions (NGJs) exhibit nonvolatile resistance switching phenomena even though the NGJs do not have any memristive medium between electrodes. They found a large change in the resistance with a ratio of up to $10^{5}$ depending on the waveform of the applied voltage, which was explained by the change in the gap distance between the electrodes. The resistive switching occurs on Pd, $\mathrm{Pt}$, and Ta electrodes as well as Au electrodes. ${ }^{8}$ Furthermore, it is also observed in the NGJs made of silicon electrodes, which demonstrates that the NGJ is compatible with semiconductor process. ${ }^{9}$ Therefore, the NGJ is expected as a building block of the next-generation memory systems. However, the NDR characteristics and switching phenomena have only been observed on the NGJs with relatively wide electrode pairs which may contain multiple junctions until recently. ${ }^{10}$ Suga et al. ${ }^{10}$ recently reported that the NGJ made by controlled break of a 45 -nm-width nanowire also shows NDR characteristics and switching phenomena. However, they found that the active tunneling area in the device is still wide and the electron tunneling is occurring at multiple sites. It would be important to fabricate narrower and thinner NGJ so that the electron tunneling occurs at a single asperity for elucidation of the switching mechanisms of the NGJ and from the viewpoint of the reduction in the operating current and miniaturization of the device as well.

${ }^{\text {a)} E l e c t r o n i c ~ m a i l: ~ h-y a m a d a @ k u e e . k y o t o-u . a c . j p . ~}$
In this study, we fabricated a single tunneling junction with a nanometer-scale gap between Pt electrodes by an electron-beam (EB) lithography process, and investigated electrical properties, resistive switching characteristics, and structural changes around the gap between electrodes by atomic force microscopy (AFM).

A single metallic tunneling junction device was fabricated by the fabrication processes as shown in Fig. 1(a). First, access pads consisting of $3 \mathrm{~nm} \mathrm{Cr}$ and $100 \mathrm{~nm}$ Au were fabricated on Si substrate covered with 300-nm-thick thermally oxidized layer using conventional photolithography process. Second, we fabricated 5-nm-thick Pt electrodes with nanometer-scale gap with the EB lithography process em-

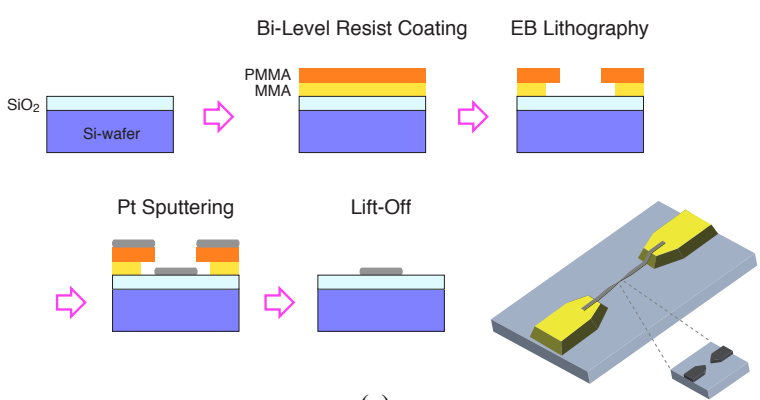

(a)

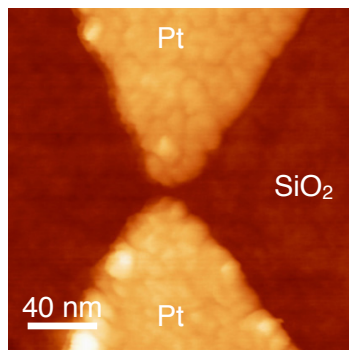

(b)

FIG. 1. (Color online) (a) Schematic of the fabrication process of single tunneling junction between Pt electrodes with a nanometer-scale gap. (b) Topographic AFM image of fabricated narrow Pt electrodes with a nanometer-scale gap. 
ploying a bilayer resist film. We spun methyl methacrylate (MMA) film as a bottom layer, and poly-MMA (PMMA) film as a top layer. After baking the resist film, electrodes with a nanometer-scale gap were defined between the $\mathrm{Au} / \mathrm{Cr}$ access pads by the EB. After development, an overhang structure was obtained because MMA dissolves faster than PMMA, which prevents ragged edge formation after lift-off process. We deposited ultrathin Pt film with a thickness of $5 \mathrm{~nm}$ by rf sputtering. We employed Pt for this study since we already established ultrathin and continuous Pt film by $\mathrm{rf}$ sputtering. ${ }^{11}$ Finally the resist film was lifted off by agitation in removing agent (Microposit: Remover 1165).

Figure 1(b) shows a topographic AFM image taken on the area around the electrode gap of a fabricated NGJ device. We used a commercially available AFM instrument (JEOL: JSPM-5200) and a Si cantilever (Olympus: OMCL-AC160) whose nominal spring constant and resonance frequency were $42 \mathrm{~N} / \mathrm{m}$ and $300 \mathrm{kHz}$, respectively. The image was taken with the intermittent contact mode. The gap distance between electrodes measured from the cross-sectional line profile of the image was about $5 \mathrm{~nm}$, which might be slightly smaller than the actual gap distance because the tip radius was about $5 \mathrm{~nm}$.

We measured I-V characteristics of the Pt NGJ in vacuum whose base pressure was about $10^{-2} \mathrm{~Pa}$ at room temperature using a semiconductor characterization system (Keithley: 4200-SCS). The resistance of the device after fabrication was above the measurement limit. Then we swept the current between the gap from 0 to $10 \mu \mathrm{A}$ with a rate of $60 \mathrm{nA} / \mathrm{s}$. After the sweep, the device showed a clear NDR characteristics and switching phenomena, therefore, we call this procedure as "forming." The I-V characteristics of the device are shown in Fig. 2(a). The thin line is the result obtained when the voltage was swept from 0 to $8 \mathrm{~V}$ at $1 \mathrm{~V} / \mathrm{s}$ and the thick line is that when the voltage was swept from 8 to $0 \mathrm{~V}$ at $1 \mathrm{~V} / \mathrm{s}$. When the voltage was swept from 0 to $8 \mathrm{~V}$, the current reached its maximum at $6.5 \mathrm{~V}$ and then drastically decreased. On the other hand, when the voltage was decreased from 8 to $0 \mathrm{~V}$, the current reached its maximum at $6.5 \mathrm{~V}$ and then decreased. These I-V curves clearly showed NDR characteristics and a hysteresis behavior in the voltage range from 4 to $6.5 \mathrm{~V}$. These NDR characteristics were measured with a high reproducibility, and they are very similar to those of NGJs with multiple tunneling junctions. ${ }^{8,10}$ In the $\mathrm{I}-\mathrm{V}$ characteristics, the current fluctuated in the voltage range from 6.5 to $8 \mathrm{~V}$. This probably suggests the migration of $\mathrm{Pt}$ atoms, which is induced by a large current.

Figure 2(b) shows the AFM image of the same area with Fig. 1(b) after sweeping the current. Apparent gap distance in Fig. 2(b) is approximately $1 \mathrm{~nm}$. We can recognize the structural change at the anode after the forming process. The height of the anode changed from 5 to $13 \mathrm{~nm}$ after the forming while that of the cathode was maintained at $5 \mathrm{~nm}$, which implies that the anode grew toward the cathode and the gap distance eventually narrowed. ${ }^{12}$

It should be noted that we also fabricated the same structures with $\mathrm{Au} / \mathrm{Cr}$ electrodes whose thickness were about $13 \mathrm{~nm}$, and found that they also showed the NDR characteristics. In the case of the $\mathrm{Au} / \mathrm{Cr}$ gap, the characteristic voltage at which the voltage shows the maximum current was about $4 \mathrm{~V}$, which was lower that in the case of the Pt gap $(6.5 \mathrm{~V})$. However, the structural change at the gap was devastating and it is difficult to fabricate ultrathin and continuous $\mathrm{Au}$
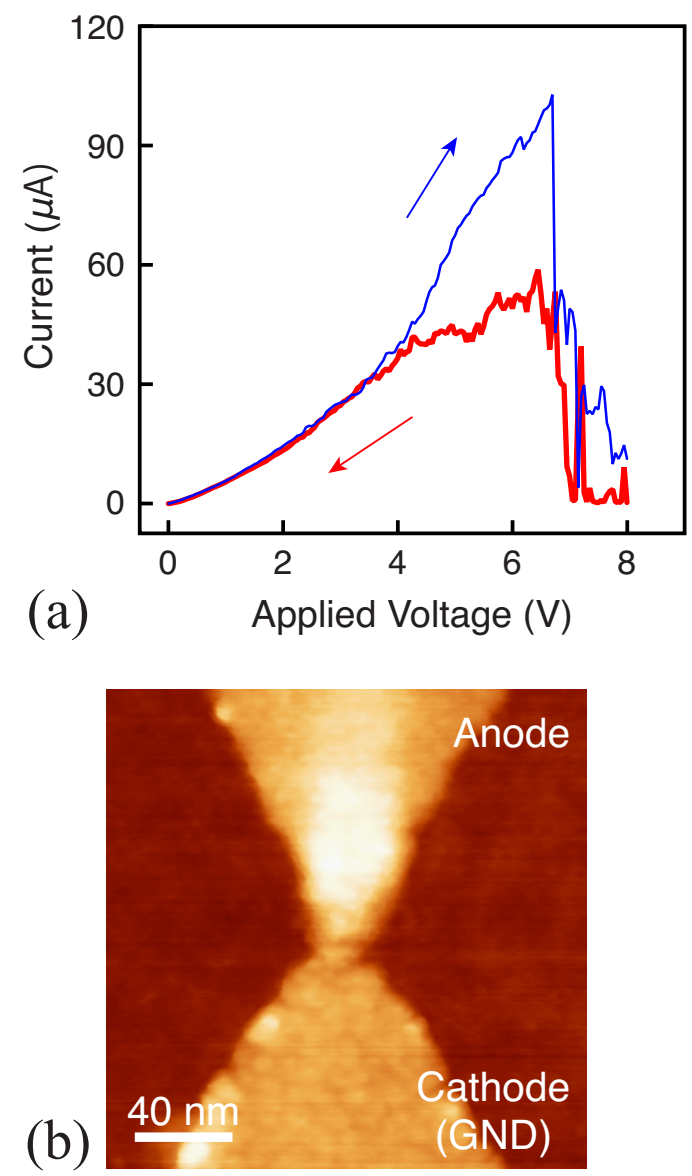

FIG. 2. (Color online) (a) I-V characteristics of narrow Pt electrodes after the current sweep. (b) Topographic AFM image of the same electrodes shown in Fig. 1(b), taken after "forming."

electrodes whose thickness is lower than $5 \mathrm{~nm}$ at the moment, therefore, we considered $\mathrm{Pt}$ is suitable for the purpose of the study.

It has been reported that the resistance of the metallic electrodes with a nanometer-scale gap shows the memory effect by applying voltage of different waveforms. ${ }^{6}$ When the applied voltage is rapidly swept from a relatively high value such as $8 \mathrm{~V}$ to a low value such as $0 \mathrm{~V}$ (off-pulse), the resistance of the NGJ is set at a high resistance state. When the applied voltage was slowly and continuously swept from a relatively high value to a low value, by contrast, the resistance of the NGJ becomes a low value (on-pulse). This is because the tunneling gap distance changes as the electrode gap distance changes depending on the waveform of the applied voltage. Figure 3(a) shows the resistive hysteresis characteristics (R-V plot) of the Pt NGJ device. Before this measurement, a voltage was swept from 8 to $0 \mathrm{~V}$ at a rate of 200 $\mathrm{kV} / \mathrm{s}$ so that the resistance between electrodes was set at the high resistance state. The thin curve is the result obtained when the applied voltage was changed from 0 to $8 \mathrm{~V}$ at 1 $\mathrm{V} / \mathrm{s}$, and the thick curve is that when the applied voltage was changed from 8 to $0 \mathrm{~V}$ at $1 \mathrm{~V} / \mathrm{s}$. The resistive hysteresis was observed between 0 and $3 \mathrm{~V}$ and the resistance ratio differed by nearly four orders of magnitude. The resistance considerably decreased at about $3 \mathrm{~V}$ when the applied voltage changed from 0 to $8 \mathrm{~V}$, which indicates that the tunneling gap distance suddenly reduced at that voltage. Moreover, the R-V curves varied discontinuously at $5.2 \mathrm{~V}$ on the blue curve and at $6 \mathrm{~V}$ on the red one. These phenomena suggest that the 

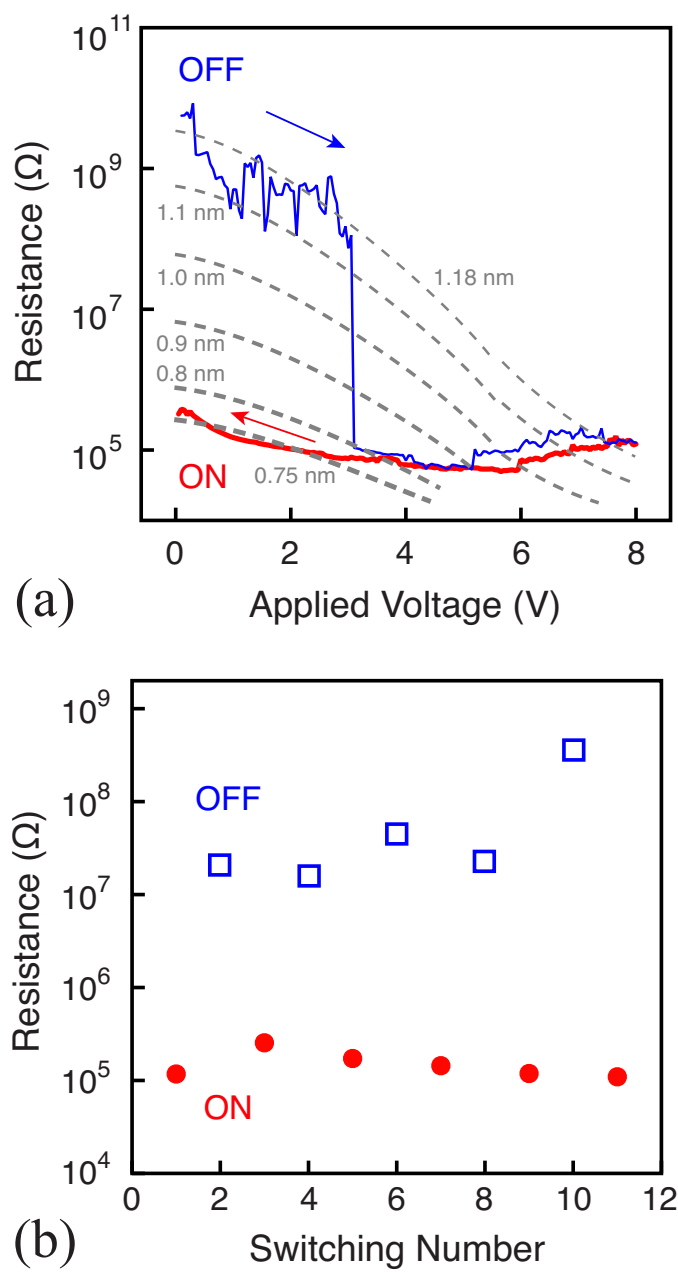

FIG. 3. (Color online) (a) I-V characteristics of narrow Pt electrodes measured after the resistance of the electrodes was set at a high resistance state. Broken curves show theoretical tunneling characteristics (Simmons' tunneling formula). (b) Repetitive resistive switching characteristics of narrow $\mathrm{Pt}$ electrodes.

change in the gap distance is not continuous but it is discontinuous.

The broken curves show the theoretical tunneling characteristics described by Eq. (50) in Ref. 13 for a work function of Pt $[5.7 \mathrm{eV}$ (Ref. 14)] and a tunneling area of $100 \mathrm{~nm}^{2}$, estimated by the apparent width of the junction from Fig. 2(b) $(20 \mathrm{~nm})$ and the Pt film thickness $(5 \mathrm{~nm})$. The measured tunneling characteristics between 0 and $3 \mathrm{~V}$ matched to the model, which strongly suggests that the device consists of a single-asperity tunneling junction. On the other hand, Suga et al. also fitted the tunneling characteristics in Ref. 10 assuming unusually small work function (0.6 $\mathrm{eV})$. This is probably because they fitted the characteristics of the multiple tunneling junctions to the single tunneling junction model. As shown in Fig. 3(a), the gap distances of the high and low resistance states were estimated as about $1.18 \mathrm{~nm}$ and $0.75 \mathrm{~nm}$, respectively. The figure illustrates the mechanisms of the resistive switching effects. When the applied voltage exceeds $3 \mathrm{~V}$, the gap distance suddenly reduces down to about $0.8 \mathrm{~nm}$, and then the gap distance gradually increases according to the increase in the applied voltage. When the applied voltage is disabled very quickly in this range, the gap distance is maintained at a larger distance, which sets the resistance at a high resistance state. When the applied voltage is disabled at $8 \mathrm{~V}$, the gap distance reaches about $1.2 \mathrm{~nm}$, giving a high resistance value. On the other hand, when the applied voltage is swept slowly down to $0 \mathrm{~V}$, the gap distance becomes about $0.8 \mathrm{~nm}$, giving a low resistance value.

Figure 3(b) shows the resistive switching characteristics of the Pt NGJ. The resistance was measured at $0.2 \mathrm{~V}$ after every resistive switching from off-state to on-state or from on-state to off-state. To change the resistance of single NGJ from on-state to off-state, the applied voltage was rapidly changed from 8 to $0 \mathrm{~V}$ at $200 \mathrm{kV} / \mathrm{s}$ In contrast, the applied voltage was continuously changed from 8 to $0 \mathrm{~V}$ at $2 \mathrm{~V} / \mathrm{s}$ to change the resistance from off-state to on-state. The resistance ratio differed by more than two orders of magnitude.

In conclusion, we fabricated a single asperity Pt NGJ whose thickness was about $5 \mathrm{~nm}$ by the EB lithography with a bilayer resist, and investigated the electrical characteristics. The NGJ having a single-asperity tunneling junction showed the NDR characteristic the resistive switching effects, which had only been reported for the devices with multiple tunneling junctions. AFM observations before and after the current sweep showed that the gap distance was narrowed after the current sweep. The tunneling area and the gap distance for on/off-state were quantitatively estimated by the fitting the measured characteristics to the simple model as $100 \mathrm{~nm}^{2}$ and $0.8 / 1.2 \mathrm{~nm}$, respectively. The resistance ratio differing by more than two orders of magnitude was measured routinely. However, the details of the structural change in the gap with the resistive switching have not been clarified yet. We consider that AFM observations before and after the operation of NGJ as a memory device might give us any insight on the operating mechanisms. The results demonstrate that the resistive switching effect occurs at the very ends of narrow electrode pairs, therefore, the NGJ is suitable for further miniaturization and it is a promising candidate for a building block of the future nonvolatile memory systems.

This work was supported by the New Energy and Industrial Technology Development Organization of Japan. We acknowledge Kyoto-Advanced Nanotechnology Network Project of the Ministry of Education, Culture, Sports, Science and Technology of Japan for technical support.

${ }^{1}$ M. Wuttig and N. Yamada, Nature Mater. 6, 824 (2007), and references therein.

${ }^{2}$ A. Sawa, Mater. Today 11, 28 (2008), and references therein.

${ }^{3}$ K. Terabe, T. Hasegawa, T. Nakayama, and M. Aono, Nature (London) 433, 47 (2005)

${ }^{4}$ R. Waser and M. Aono, Nature Mater. 6, 833 (2007).

${ }^{5}$ C. P. Collier, E. W. Wong, M. Belohradsky, F. M. Raymo, J. F. Stoddart, P. J. Kuekes, R. S. Williams, and J. R. Heath, Science 285, 391 (1999).

${ }^{6}$ Y. Naitoh, M. Horikawa, H. Abe, and T. Shimizu, Nanotechnology 17, 5669 (2006)

${ }^{7}$ Y. Naitoh, M. Horikawa, and T. Shimizu, Jpn. J. Appl. Phys. 47, 400 (2008).

${ }^{8}$ S. Furuta, T. Takahashi, Y. Naitoh, M. Horikawa, T. Shimizu, and M. Ono, Jpn. J. Appl. Phys. 47, 1806 (2008).

${ }^{9}$ Y. Naitoh, Y. Morita, M. Horikawa, H. Suga, and T. Shimizu, Appl. Phys. Express 1, 103001 (2008).

${ }^{10}$ H. Suga, M. Horikawa, S. Okada, H. Miyazaki, K. Tsukagoshi, T. Shmidzu, and Y. Naitoh, Appl. Phys. Lett. 97, 073118 (2010).

${ }^{11}$ T. Miyazaki, K. Kobayashi, K. Ishida, T. Horiuchi, H. Yamada, and K. Matsushige, Jpn. J. Appl. Phys., Part 1 42, 4173 (2003).

${ }^{12}$ Y. Tomoda, K. Takahashi, M. Hanada, W. Kume, and J. Shirakashi, J. Vac. Sci. Technol. B 27, 813 (2009).

${ }^{13}$ J. G. Simmons, J. Appl. Phys. 34, 1793 (1963).

${ }^{14}$ Handbook of Physics, edited by W. Benenson, J. W. Harris, H. Stöcker, and H. Lutz (Springer, New York, 2002). 